Supporting Information for

\title{
Charge Separation by Imidazole and Sulfonic Acid Functionalized Covalent Organic Frameworks for Enhanced Proton Conductivity
}

Shuyang Bian, ${ }^{\ddagger a}$ Kun Zhang, ${ }^{\ddagger a}$ Yuxiang Wang, ${ }^{\text {a } Z i y a ~ L i u, ~}{ }^{\mathrm{a}}$ Guixiang Wang, ${ }^{\text {*a }}$ Xinzhu Jiang, ${ }^{\text {a }}$ Yaoyao Pan, ${ }^{a}$ Bingqing Xu, ${ }^{* a}$ Guoji Huang, ${ }^{a}$ Gen Zhang*a

${ }^{a}$ Key Laboratory for Soft Chemistry and Functional Materials of Ministry of Education, School of Chemistry and Chemical Engineering, Nanjing University of Science and Technology, Nanjing, Jiangsu 210094, China.

${ }^{\ddagger}$ S. B. and K. Z. contributed equally to this work.

This work dedicated to Professor Susumu Kitagawa on the occasion of his 70th birthday.

*Correspondence and requests for materials to be addressed to G.W. Email: wanggx1028@163.com and G.Z. Email: zhanggen@njust.edu.cn 


\section{Materials}

All chemicals and solvents used in the syntheses are reagent grade and used without further purification. 1,3,5-Benzenetricarboxaldehyde and 2,5diethoxyterephthalohydrazide were purchased from Extension and Tensus Biotech, respectively. $\mathrm{NH}_{4} \mathrm{Br}$, 1,3-Propane sultone and Imidazole were purchased from Nanjing Wanqing Chemical Glassware Instrument Co., Ltd.. All the solvents such as acetic acid, dichloromethane, acetone, petroleum ether (PE) was purchased from Sinopharm.

\section{Measurements}

Powder X-ray diffraction (PXRD): PXRD patterns were collected on a Bruker D8 Advance diffractometer using $\mathrm{Cu} \mathrm{K} \alpha$ radiation.

Fourier transform infrared (FT-IR): FT-IR spectrum was measured on a Thermo Fisher Scientific Optics NICOLETIS10 FT-IR spectrometer with Universal ATR accessory between the ranges of 4000 to $525 \mathrm{~cm}^{-1}$.

Solution nuclear magnetic resonance (NMR): Liquid state ${ }^{1} \mathrm{H}$ nuclear magnetic resonance spectra was collected on a Bruker Avance III instrument with AS500 magnet equipped with a cryoprobe $(500 \mathrm{MHz})$.

High-resolution mass spectrometry (HRMS): HRMS mass spectra were collected on a Baird Acquity UPLC/XEVO G2-XS QTOF using $\mathrm{CHCl3}$ as a solvent.

Elemental analysis: The elemental analysis was conducted with the elemental analysizer FLASHSMART.

Scanning electron microscope (SEM): SEM images were collected using a JSMIT500HR system.

Gas adsorption: Gas adsorption measurement N2 adsorption and desorption measurements were performed at $77 \mathrm{~K}$ using ASAP 2020, Micromeritics Instrument Corp, USA. Pore size distributions and pore volumes were derived from the adsorption isotherms.

Thermogravimetric analysis (TGA): TGA was performed using a TGA/SDTA851E, Mettler Toledo, under flowing $\mathrm{N}_{2}(60 \mathrm{~mL} \mathrm{~min}-1)$ with $10 \mathrm{~K} \mathrm{~min}^{-1}$ ramp rate. Samples were heated in a Platinum pan from $50{ }^{\circ} \mathrm{C}$ to $900{ }^{\circ} \mathrm{C}$.

Water vapor adsorption: water vapor adsorption and desorption measurement Use Microphone TriStar II 3020 at $25^{\circ} \mathrm{C}$ 


\section{Synthesis of $\mathbf{P}_{3}\left(\mathrm{C} 3-\mathrm{Im}-\mathrm{NHNH}_{2}\right)^{[1-2]}$}
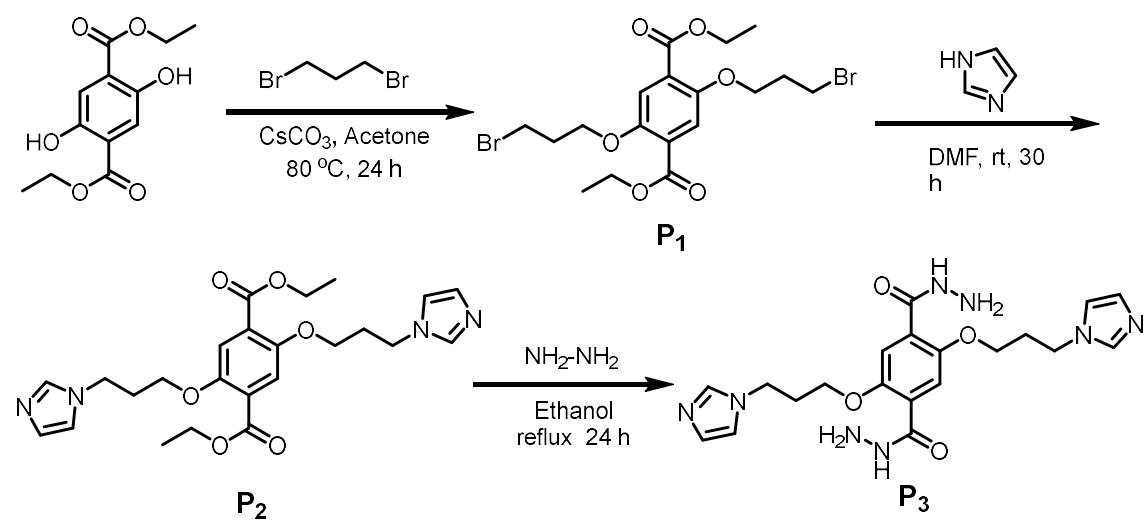

\section{Synthesis of $\mathbf{P}_{1}$}

Diethyl 2,5-dihydroxyterephthalate $(2.54 \mathrm{~g}, 10 \mathrm{mmol})$, and cesium carbonate (4.89 $\mathrm{g}, 15 \mathrm{mmol}$ ) were added to the dry acetone solution under $\mathrm{N}_{2}$ atmosphere. The reaction was refluxed at $80^{\circ} \mathrm{C}$ for 24 hours. The obtained product was filtered and extracted with dichloromethane. The organic layer was dried over anhydrous $\mathrm{Na}_{2} \mathrm{SO}_{4}$ and concentrated in vacuum. The crude product was purified by a flash chromatography column (dichloromethane: petroleum ether $=5: 1)$ to obtain a white solid product $\left(\mathbf{P}_{1}\right)$ after dry under high vacuum overnight. Yield 45\%. ${ }^{1} \mathrm{H}$ NMR (500 MHz, $\left.\mathrm{CDCl}_{3}\right) \delta(\mathrm{ppm}) 7.40$ $(2 \mathrm{H}, \mathrm{s}), 4.37(4 \mathrm{H}, \mathrm{m}), 4.16(4 \mathrm{H}, \mathrm{m}), 3.66(4 \mathrm{H}, \mathrm{m}), 2.34(4 \mathrm{H}, \mathrm{m})), 1.39(6 \mathrm{H}, \mathrm{m}) .{ }^{13} \mathrm{C} \mathrm{NMR}$ $\left(500 \mathrm{MHz}, \mathrm{CDCl}_{3}\right) \delta(\mathrm{ppm}) 165.31,151.44,124.44,116.67,66.93,61.11,32.35,29.93$, 14.19.

\section{Synthesis of $\mathbf{P}_{2}$}

Imidazole $(2.72 \mathrm{~g}, 40 \mathrm{mmol})$ was added to a solution of diethyl 2,5-bis(3bromopropoxy)terephthalate $\left(\mathbf{P}_{1}\right)(4.96 \mathrm{~g}, 10 \mathrm{mmol})$ in DMF $(50 \mathrm{~mL})$. The mixture was stirred for $30 \mathrm{~h}$ at room temperature, then a saturated $\mathrm{NaCl}$ aqueous solution $(50 \mathrm{~mL})$ and EtOAc $(50 \mathrm{~mL})$ were added. The resulted organic phase suspended was separated, and washed with saturated $\mathrm{NaCl}$ aqueous solution $(50 \mathrm{~mL})$ three times, and dried over $\mathrm{MgSO}_{4}$. The crude product was purified by flash chromatographic column (DCM:MeOH=95:5)to obtain a white solid product $\left(\mathbf{P}_{2}\right)$ after dry under high vacuum overnight. Yield 48\%. ${ }^{1} \mathrm{H}$ NMR $\left(500 \mathrm{MHz}, \mathrm{CDCl}_{3}\right): \delta(\mathrm{ppm}) 7.50(2 \mathrm{H}, \mathrm{s}), 7.30(2 \mathrm{H}, \mathrm{s})$, $7.03(2 \mathrm{H}, \mathrm{s}), 6.93(2 \mathrm{H}, \mathrm{s}), 4.38(4 \mathrm{H}, \mathrm{m}), 4.28(4 \mathrm{H}, \mathrm{m}), 3.92(4 \mathrm{H}, \mathrm{m}), 2.22(4 \mathrm{H}, \mathrm{m}), 1.38$ $(6 \mathrm{H}, \mathrm{m}),{ }^{13} \mathrm{C}$ NMR $\left(500 \mathrm{MHz}, \mathrm{CDCl}_{3}\right) \delta(\mathrm{ppm}) 165.10,151.53,137.47,129.65,124.69$, 118.96, 116.53, 65.12, 42.90, 30.61, 14.28. HR-MS (EI): calcd for $\mathrm{C}_{24} \mathrm{H}_{30} \mathrm{~N}_{4} \mathrm{O}_{6} 475.53$; 
found 474.23187 .

\section{Synthesis of $P_{3}$}

$2.35 \mathrm{~g}$ ( $5 \mathrm{mmol}$ ) of $\mathbf{P}_{2}$ was dissolved in $30 \mathrm{ml}$ ethanol and $2 \mathrm{ml}$ hydrazine hydrate, then heated to $90{ }^{\circ} \mathrm{C}$. The mixture was stirred and heated to reflux for 24 hours, after the reaction over. The crude product was washed with petroleum ether, and the crude product was purified to obtain a white solid product $\left(\mathbf{P}_{3}\right)$ after dry under high vacuum overnight. Yield 88\%. ${ }^{1} \mathrm{H}$ NMR (500 MHz, DMSO): $\delta(\mathrm{ppm}) 9.34(2 \mathrm{H}, \mathrm{s}), 7.77(2 \mathrm{H}, \mathrm{s})$, 7.27 (2H,d), 7.24 (2H,s),6.97 (2H,d), 4.19 (4H,s), 3.97 (4H,m), 2.21(4H,m). HR-MS (EI): calcd for $\mathrm{C}_{20} \mathrm{H}_{26} \mathrm{~N}_{8} \mathrm{O}_{4} 442.48$; found 443.21454 .

\section{Elemental analysis of composites}

Table S1. Elemental analysis of composites

\begin{tabular}{cccc}
\hline & $\mathrm{C}(\%)$ & $\mathrm{H}(\%)$ & $\mathrm{N}(\%)$ \\
\hline COF-Im & 45.29 & 4.77 & 14.77 \\
$\mathrm{NH}_{4} \mathrm{Br} @$ COF-Im $^{+}$ & 44.81 & 4.91 & 15.46 \\
COF-Im ${ }^{+}$SO3 $^{-}$ & 39.18 & 4.97 & 9.89 \\
$\mathrm{NH}_{4} \mathrm{Br} @ \mathrm{COF}^{-} \mathrm{Im}^{+}$-SO3 $^{-}$ & 39.22 & 4.74 & 12.12 \\
\hline
\end{tabular}

\section{Structural Simulation for COF-Im ${ }^{[3]}$}

Table S2. Fractional atomic coordinates for simulated COF-Im powder.

\begin{tabular}{|c|c|c|c|}
\hline \multicolumn{4}{|l|}{ Space group: $P 3$} \\
\hline \multicolumn{2}{|c|}{ 2D hexagonal; $\mathrm{a}=\mathrm{b}=28.91 \AA, \mathrm{c}=4.20 \AA ; \alpha=\beta=90^{\circ}, \gamma=120^{\circ}$} \\
\hline Atom & $\mathbf{x}$ & $\mathbf{y}$ & $\mathbf{z}$ \\
\hline $\mathrm{C} 1$ & 0.10824 & 0.27058 & -0.22874 \\
\hline $\mathrm{C} 2$ & 0.16316 & 0.28895 & -0.2561 \\
\hline $\mathrm{C} 3$ & 0.20207 & 0.33831 & -0.12836 \\
\hline $\mathrm{C} 4$ & 0.18512 & 0.37036 & 0.02833 \\
\hline $\mathrm{C} 5$ & 0.13025 & 0.35135 & 0.06794 \\
\hline $\mathrm{C} 6$ & 0.09141 & 0.30137 & -0.05517 \\
\hline
\end{tabular}




\begin{tabular}{|c|c|c|c|}
\hline $\mathrm{C} 7$ & 0.2248 & 0.42425 & 0.14879 \\
\hline O8 & 0.26757 & 0.43209 & 0.25203 \\
\hline C9 & 0.06884 & 0.21716 & -0.36286 \\
\hline $\mathrm{O} 10$ & 0.02366 & 0.19292 & -0.25479 \\
\hline N11 & 0.04925 & 0.13993 & -0.67597 \\
\hline N12 & 0.08288 & 0.19386 & -0.59883 \\
\hline N13 & 0.25546 & 0.51795 & 0.20318 \\
\hline N14 & 0.21466 & 0.46566 & 0.13687 \\
\hline $\mathrm{C} 15$ & 0.06624 & 0.11369 & -0.84244 \\
\hline $\mathrm{C} 16$ & 0.03216 & 0.05584 & -0.88625 \\
\hline C17 & -0.02366 & 0.032 & -0.88973 \\
\hline $\mathrm{C} 18$ & 0.24688 & 0.55788 & 0.2023 \\
\hline C19 & 0.29134 & 0.61328 & 0.2359 \\
\hline $\mathrm{C} 20$ & 0.34474 & 0.625 & 0.23792 \\
\hline $\mathrm{O} 21$ & 0.0361 & 0.27997 & -0.00601 \\
\hline $\mathrm{C} 22$ & 0.01741 & 0.31081 & 0.15681 \\
\hline $\mathrm{C} 23$ & -0.04315 & 0.27711 & 0.18973 \\
\hline $\mathrm{C} 24$ & -0.0674 & 0.3099 & 0.31821 \\
\hline $\mathrm{N} 25$ & -0.12567 & 0.27755 & 0.32734 \\
\hline $\mathrm{C} 26$ & -0.16076 & 0.28751 & 0.17514 \\
\hline $\mathrm{C} 27$ & -0.15534 & 0.23231 & 0.49207 \\
\hline $\mathrm{C} 28$ & -0.20824 & 0.21551 & 0.43379 \\
\hline N29 & -0.21194 & 0.2501 & 0.23461 \\
\hline $\mathrm{O} 30$ & 0.25767 & 0.35749 & -0.15368 \\
\hline C31 & 0.27635 & 0.32792 & -0.33328 \\
\hline C32 & 0.27104 & 0.27998 & -0.14363 \\
\hline C33 & 0.27165 & 0.2377 & -0.35913 \\
\hline N34 & 0.26347 & 0.19148 & -0.1737 \\
\hline C35 & 0.21833 & 0.14402 & -0.15167 \\
\hline
\end{tabular}




\begin{tabular}{|c|c|c|c|}
\hline $\mathrm{C} 36$ & 0.30014 & 0.18689 & -0.00078 \\
\hline N37 & 0.28005 & 0.13829 & 0.13464 \\
\hline C38 & 0.22863 & 0.11153 & 0.03921 \\
\hline H39 & 0.17548 & 0.26349 & -0.36779 \\
\hline $\mathrm{H} 40$ & 0.11823 & 0.37542 & 0.20208 \\
\hline H41 & 0.11955 & 0.21494 & -0.71507 \\
\hline H42 & 0.17813 & 0.45944 & 0.04969 \\
\hline H43 & 0.10766 & 0.13295 & -0.91671 \\
\hline H44 & -0.04204 & 0.05672 & -0.87863 \\
\hline H45 & 0.2069 & 0.55092 & 0.16023 \\
\hline H46 & 0.35379 & 0.5929 & 0.22875 \\
\hline $\mathrm{H} 47$ & 0.02812 & 0.34757 & 0.01783 \\
\hline H48 & 0.03502 & 0.32149 & 0.39913 \\
\hline H49 & -0.05278 & 0.24321 & 0.35278 \\
\hline H50 & -0.06078 & 0.2604 & -0.04606 \\
\hline H51 & -0.05531 & 0.34533 & 0.16332 \\
\hline H52 & -0.0521 & 0.32458 & 0.56125 \\
\hline H53 & -0.14942 & 0.32148 & 0.02072 \\
\hline H54 & -0.13988 & 0.21286 & 0.64237 \\
\hline H55 & -0.24213 & 0.18016 & 0.53091 \\
\hline H56 & 0.3193 & 0.35486 & -0.38718 \\
\hline H57 & 0.25581 & 0.31633 & -0.56598 \\
\hline H58 & 0.23307 & 0.26081 & -0.00831 \\
\hline H59 & 0.30463 & 0.29459 & 0.028 \\
\hline H60 & 0.31045 & 0.25496 & -0.48619 \\
\hline H61 & 0.23903 & 0.22443 & -0.5366 \\
\hline H62 & 0.18089 & 0.1337 & -0.26678 \\
\hline H63 & 0.3412 & 0.21842 & 0.02599 \\
\hline H64 & 0.20048 & 0.07075 & 0.10485 \\
\hline
\end{tabular}


Table S3. Summary of COFs based proton conductors.

\begin{tabular}{|c|c|c|c|c|}
\hline Material & $\sigma\left(\mathrm{S} \mathrm{cm}^{-1}\right)$ & RH & $\mathrm{T}\left({ }^{\circ} \mathrm{C}\right)$ & Ref. \\
\hline $\begin{array}{c}\mathrm{NH}_{4} \mathrm{Br} @ \mathrm{COF}-\mathrm{Im}^{+}- \\
\mathrm{SO}_{3}^{-}\end{array}$ & $3.69 \times 10^{-3}$ & $100 \%$ & 90 & This work \\
\hline $\begin{array}{c}\mathrm{H}_{3} \mathrm{PO}_{4} @ \text { TPB- } \\
\text { DMeTPCOF }\end{array}$ & $1.91 \times 10^{-1}$ & anhydrous & 160 & $\begin{array}{l}\text { Nat. Commun. } 2020 \text {, } \\
11,1981 .\end{array}$ \\
\hline $\mathrm{H}_{3} \mathrm{PO}_{4} @ \mathrm{Tp}-\mathrm{Azo}-\mathrm{COF}$ & $6.70 \times 10^{-5}$ & anhydrous & 67 & $\begin{array}{l}\text { J. Am. Chem. Soc. } \\
\text { 2014, 136, } 6570 .\end{array}$ \\
\hline COF-F6-H & $4.2 \times 10^{-2}$ & anhydrous & 140 & $\begin{array}{l}\text { J. Am. Chem. Soc. } \\
\text { 2020,142, } \\
\text { 14357-14364. }\end{array}$ \\
\hline $\begin{array}{c}\text { Phyticacid@TpPa- } \\
\left.\text { (SO } \mathrm{SO}_{3} \mathrm{HPy}\right)\end{array}$ & $5.00 \times 10^{-4}$ & anhydrous & 120 & $\begin{array}{l}\text { Chem. Mater. 2016, } \\
28,1489 .\end{array}$ \\
\hline $\begin{array}{c}\text { Im@TPB-DMTP- } \\
\text { COF }\end{array}$ & $4.37 \times 10^{-3}$ & anhydrous & 130 & $\begin{array}{l}\text { Nat. Mater. 2016, 15, } \\
722 .\end{array}$ \\
\hline Im@Td-PPI & $3.49 \times 10^{-4}$ & anhydrous & 90 & $\begin{array}{l}\text { J. Am. Chem. Soc. } \\
2015,137,913 .\end{array}$ \\
\hline Im@Td-PNDI & $9.04 \times 10^{-5}$ & anhydrous & 90 & $\begin{array}{l}\text { J. Am. Chem. Soc. } \\
\text { 2015, 137, } 913 .\end{array}$ \\
\hline NUS-9 (R) & $1.24 \times 10^{-2}$ & 97 & 25 & $\begin{array}{l}\text { ACS Appl. Mater. } \\
\text { Interfaces. 2016, } 8 \text {, } \\
18505 .\end{array}$ \\
\hline NUS-10 (R) & $3.96 \times 10^{-2}$ & 97 & 25 & $\begin{array}{l}\text { ACS Appl. Mater. } \\
\text { Interfaces. 2016, } 8 \text {, } \\
18505 .\end{array}$ \\
\hline BIP & $3.2 \times 10^{-2}$ & 95 & 95 & $\begin{array}{l}\text { J. Am. Chem. Soc. } \\
\text { 2019, 141, } 14950 .\end{array}$ \\
\hline PA@Tp-Azo & $9.9 \times 10^{-4}$ & 98 & 59 & $\begin{array}{l}\text { J. Am. Chem. Soc. } \\
\text { 2014, 136, } 6570 .\end{array}$ \\
\hline PA@Tp-Stb & $2.3 \times 10^{-5}$ & 98 & 59 & $\begin{array}{l}\text { J. Am. Chem. Soc. } \\
\text { 2014, 136, } 6570 .\end{array}$ \\
\hline EB-COF-Br & $2.82 \times 10^{-5}$ & 97 & 25 & $\begin{array}{l}\text { J. Am. Chem. } \\
\text { Soc. } 2016,138,5897 .\end{array}$ \\
\hline $\mathrm{H}_{3} \mathrm{PO}_{4} @ \mathrm{NKCOF}-10$ & $9.04 \times 10^{-2}$ & 90 & 80 & $\begin{array}{l}\text { Nat. Commun.2021, } \\
12,1982 .\end{array}$ \\
\hline $\begin{array}{c}\text { PEEK@Ox-DBD- } \\
\text { COF-SO }{ }_{3} \mathrm{H}\end{array}$ & $3.87 \times 10^{-3}$ & 98 & 90 & $\begin{array}{l}\text { Chem. - Eur. J.2021, } \\
27,3817 .\end{array}$ \\
\hline $\mathrm{H}_{3} \mathrm{PO}_{4} @ \mathrm{NKCOF}-4$ & $7.71 \times 10^{-2}$ & 98 & 80 & $\begin{array}{l}\text { Angew. Chem., Int. } \\
\text { Ed.2020, 59, } 3678 .\end{array}$ \\
\hline
\end{tabular}


IF-TR, water adsorption and TGA

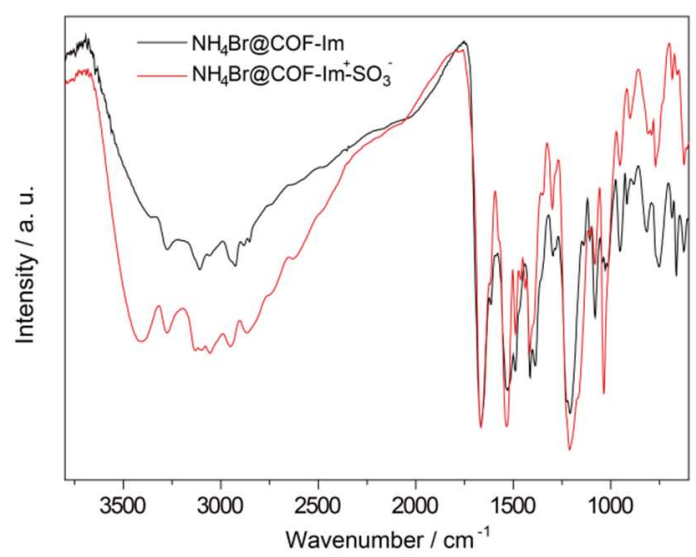

Figure S1. IR plot of $\mathrm{NH}_{4} \mathrm{Br} @ \mathrm{COF}^{-\mathrm{Im}^{+}-\mathrm{SO}^{-}}{ }^{-}$and $\mathrm{NH}_{4} \mathrm{Br} @ \mathrm{COF}-\mathrm{Im}$.

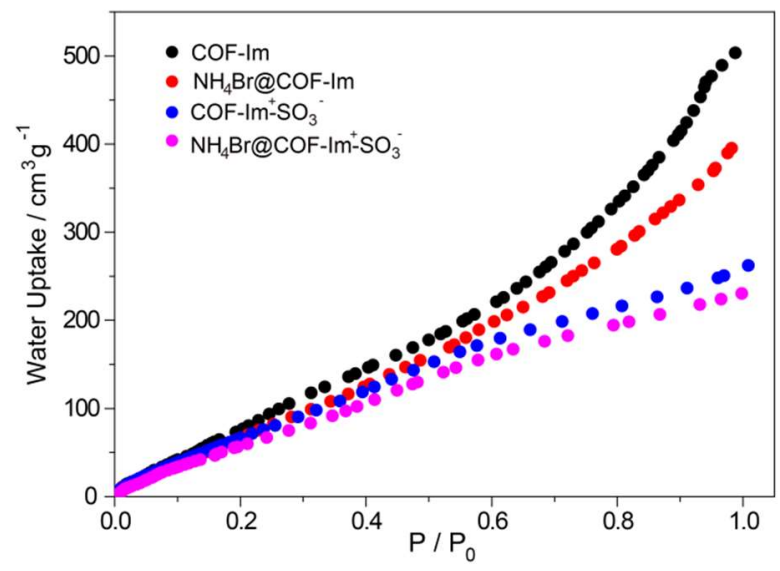

Figure S2. Water uptake of $\mathrm{NH}_{4} \mathrm{Br} @ \mathrm{COF}-\mathrm{Im}^{+}-\mathrm{SO}_{3}{ }^{-}, \mathrm{NH}_{4} \mathrm{Br} @ \mathrm{COF}-\mathrm{Im}, \mathrm{COF}-\mathrm{Im}^{+}-\mathrm{SO}_{3}{ }^{-}$ and COF-Im.

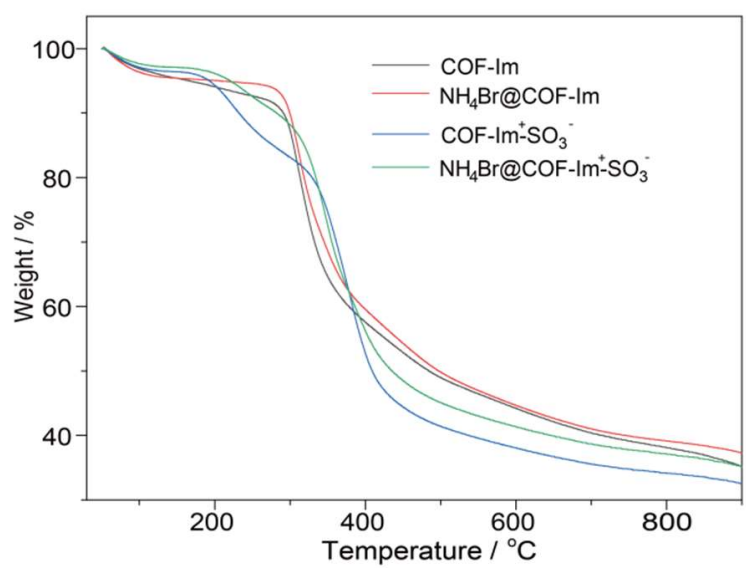

Figure S3. TGA plot of $\mathrm{NH}_{4} \mathrm{Br} @ \mathrm{COF}-\mathrm{Im}^{+}-\mathrm{SO}_{3}{ }^{-}, \mathrm{NH}_{4} \mathrm{Br} @ \mathrm{COF}-\mathrm{Im}, \mathrm{COF}-\mathrm{Im}^{+}-\mathrm{SO}_{3}{ }^{-}$ and COF-Im. 


\section{Proton conductivity of COFs}

(a)

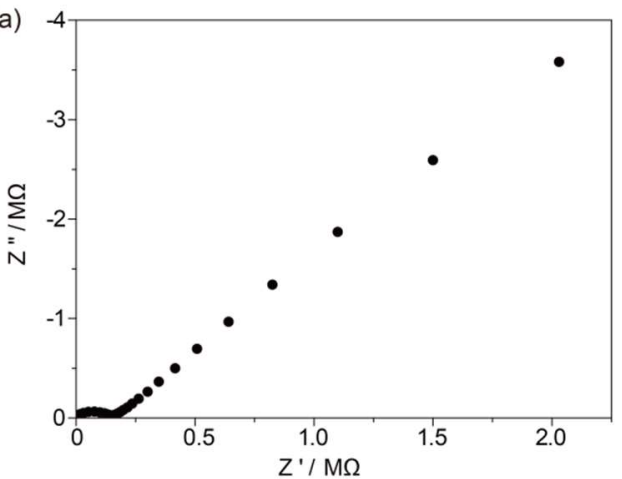

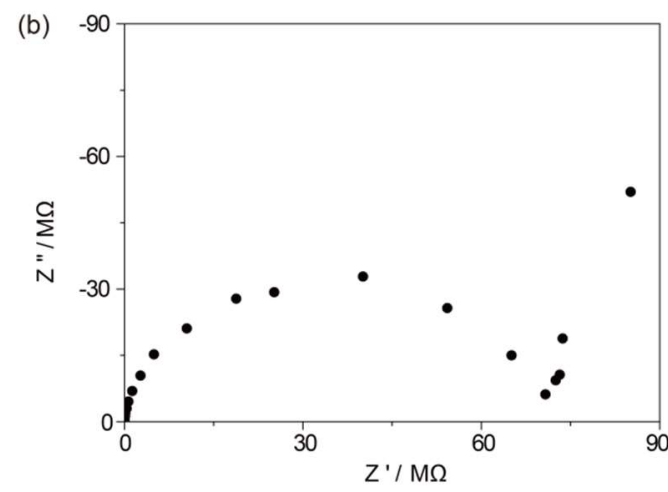

Figure S4. (a) Nyquist plot of COF-Im at $100 \% \mathrm{RH}$ and $30{ }^{\circ} \mathrm{C}$. (b) Nyquist plot of COF-Im ${ }^{+}-\mathrm{SO}_{3}{ }^{-}$ at $100 \% \mathrm{RH}$ and $30^{\circ} \mathrm{C}$.
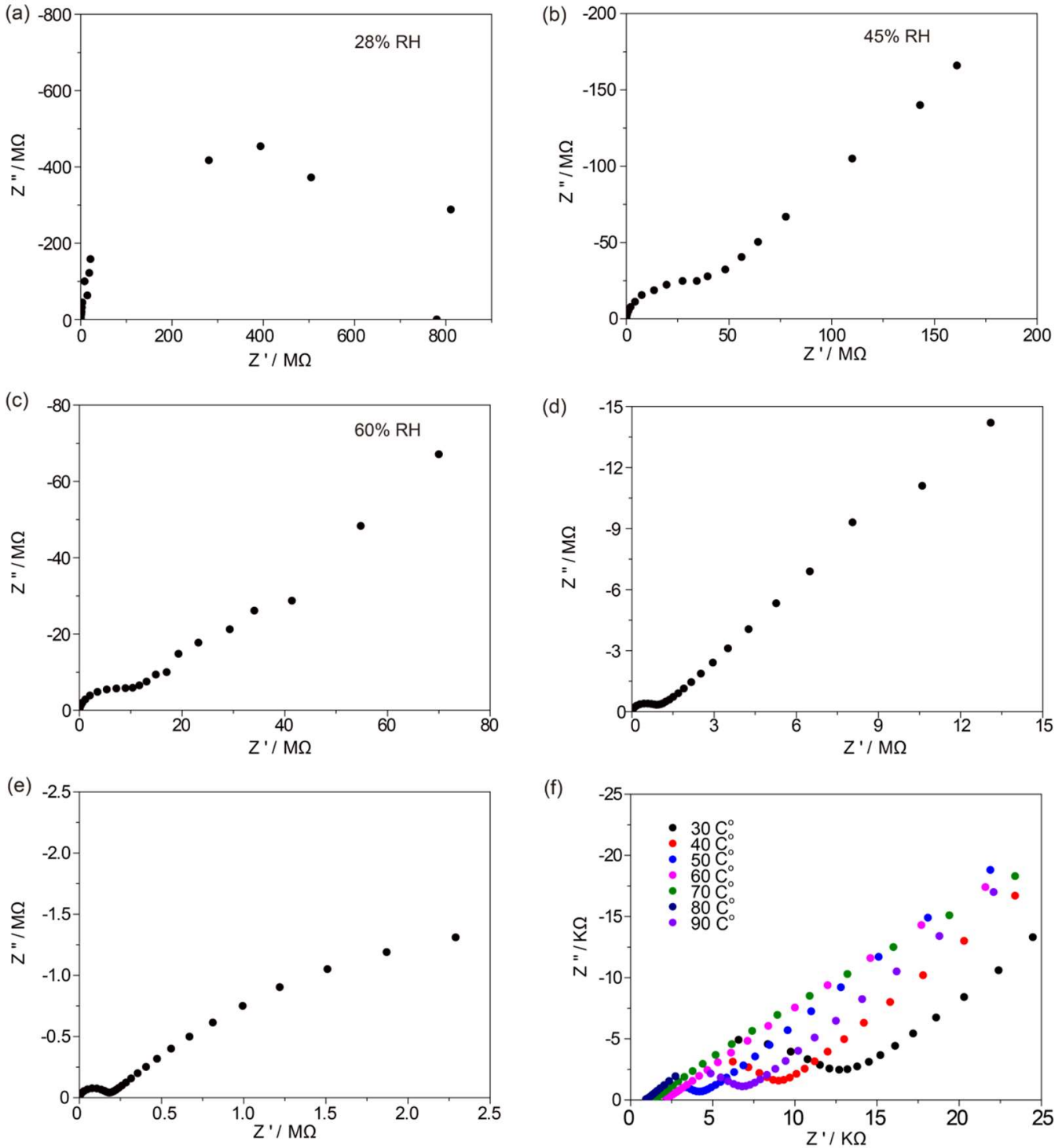

Figure S5. (a-e) Nyquist plot of $\mathrm{NH}_{4} \mathrm{Br} @$ COF-Im versus different relative humidity at $30{ }^{\circ} \mathrm{C}$. (f) Nyquist plot of $\mathrm{NH}_{4} \mathrm{Br} @$ COF-Im versus different temperature at 100\% RH. 

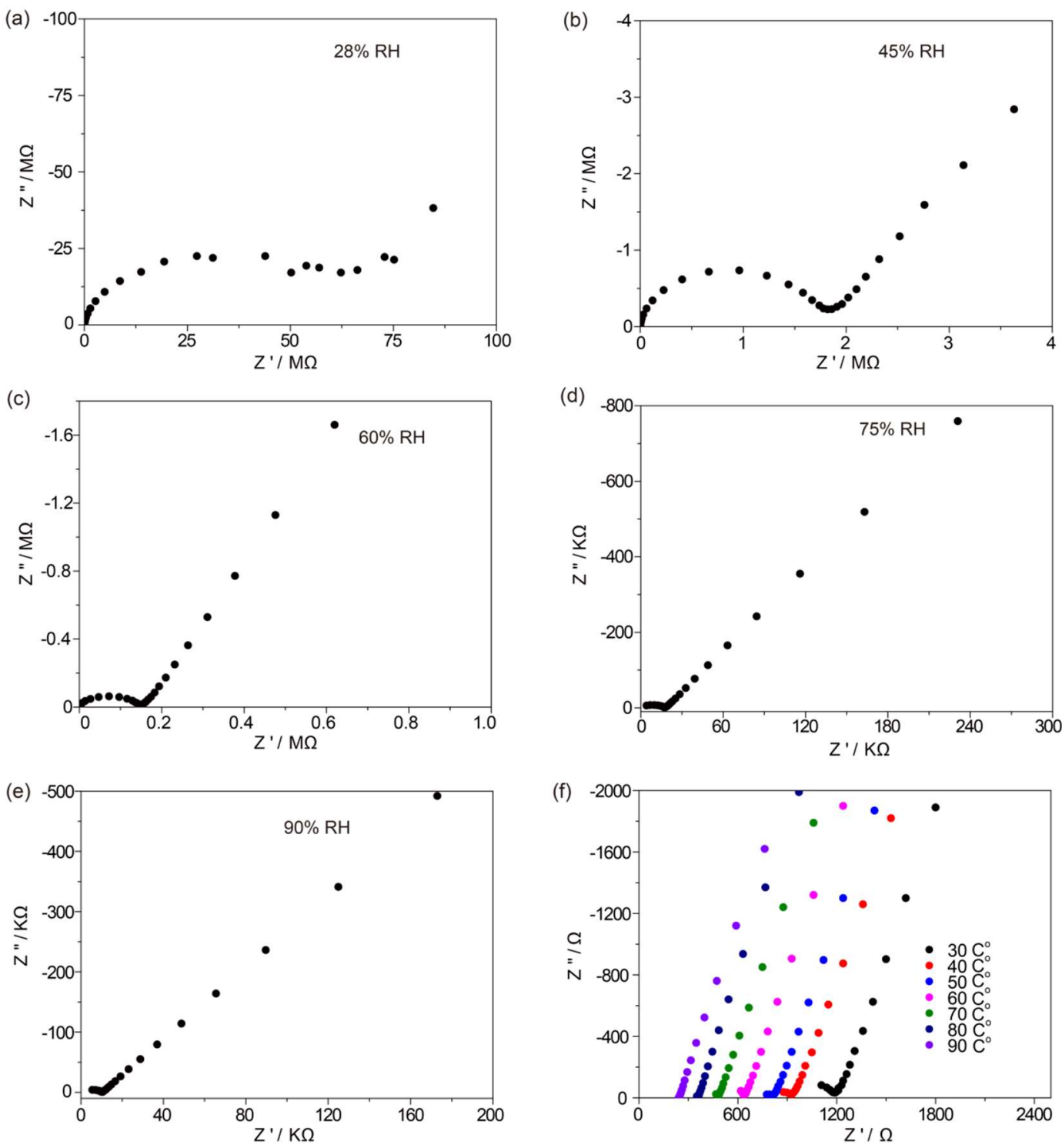

Figure S6. (a-e) Nyquist plot of $\mathrm{NH}_{4} \mathrm{Br} @ \mathrm{COF}-\mathrm{Im}^{+}-\mathrm{SO}_{3}{ }^{-}$versus different relative humidity at $30{ }^{\circ} \mathrm{C}$. (f) Nyquist plot of $\mathrm{NH}_{4} \mathrm{Br} @ \mathrm{COF}-\mathrm{Im}^{+}-\mathrm{SO}_{3}{ }^{-}$versus different temperature at $100 \% \mathrm{RH}$.

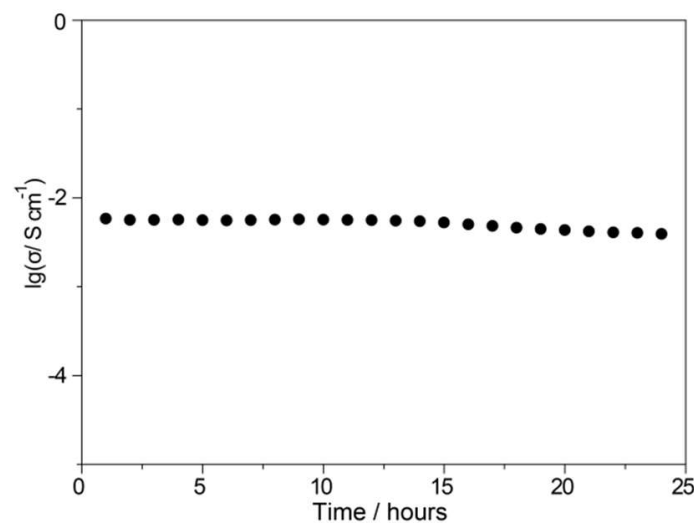

Figure S7. Long-term proton conducting durability measurement of $\mathrm{NH}_{4} \mathrm{Br} @ \mathrm{COF}-\mathrm{Im}^{+}-\mathrm{SO}_{3}{ }^{-}$at $100 \%$ RH and $90{ }^{\circ} \mathrm{C}$. 


\section{NMR spectra of monomers}
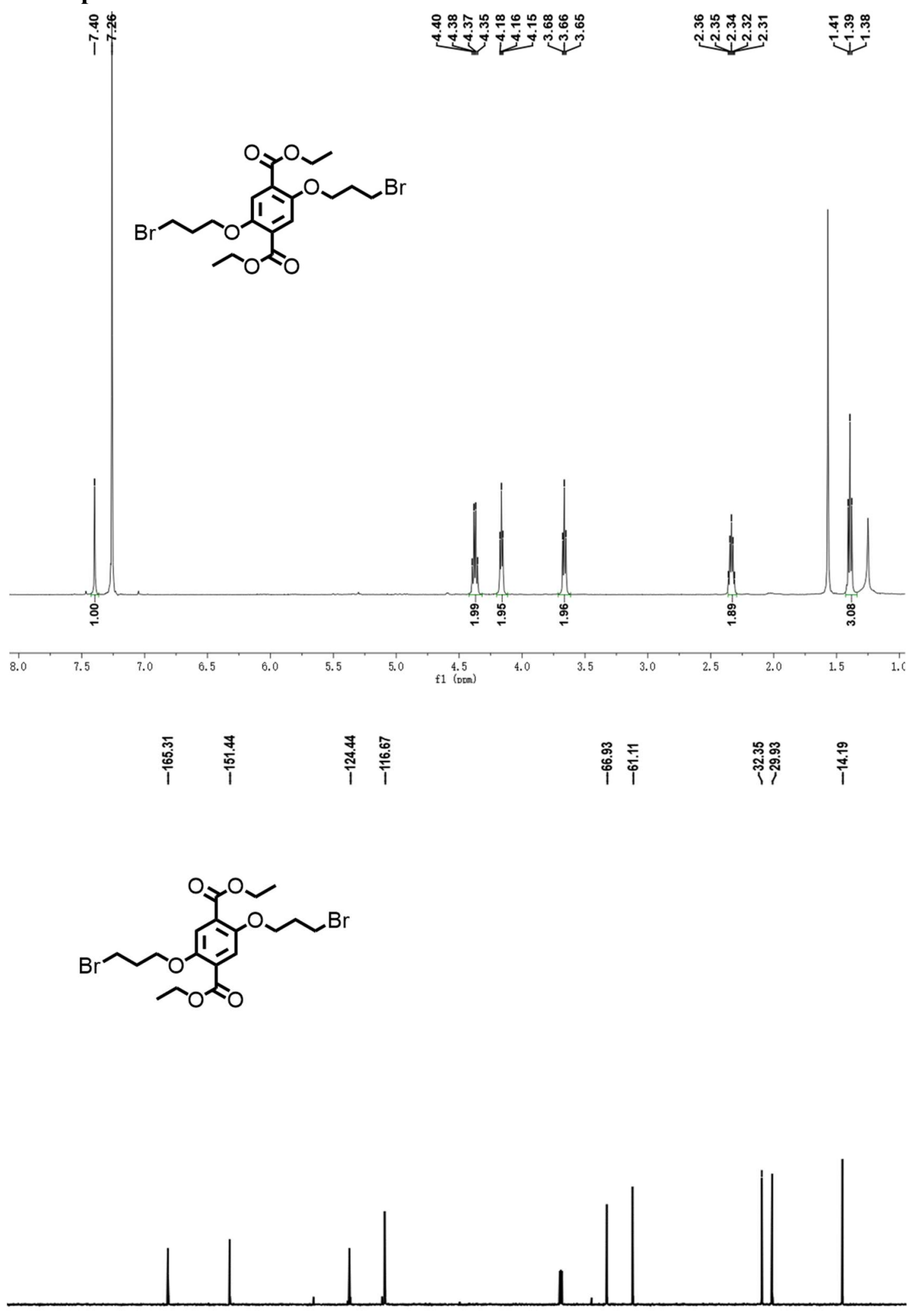

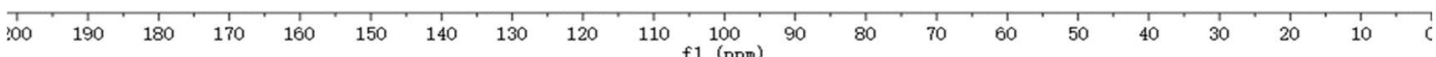

Figure S8. ${ }^{1} \mathrm{H}$ and ${ }^{13} \mathrm{C}$ date of $\mathbf{P 1}$. 
<smiles>CCOC(=O)c1cc(OCCCn2ccnc2)c(C(=O)OCC)cc1OCCCCn1ccnc1</smiles>

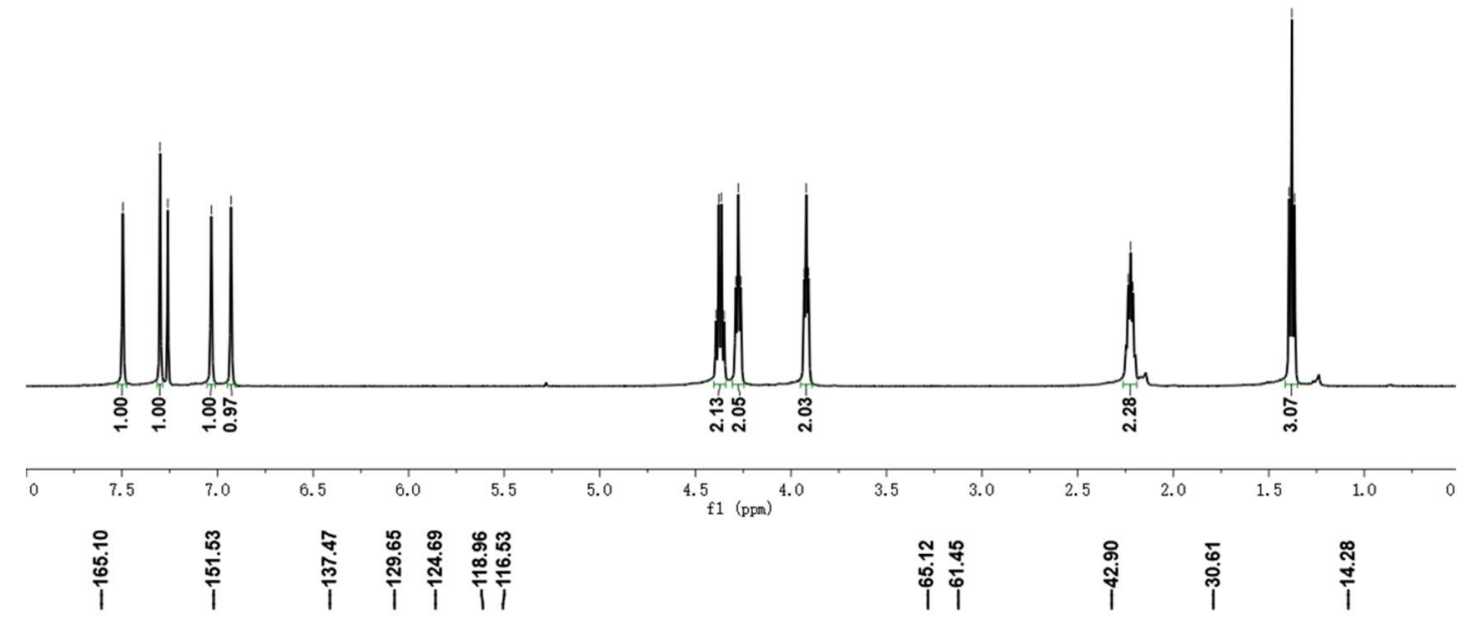<smiles>CCOC(=O)c1cc(OCCCCn2ccnc2)c(OCCCn2ccnc2)cc1C(=O)OCC</smiles>

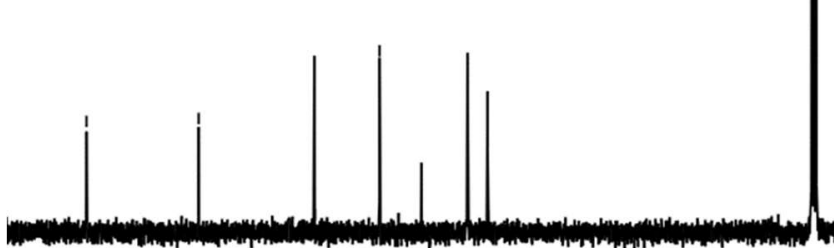

$\begin{array}{llll}170 & 160 & 150 & 140\end{array}$

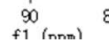

10

Figure S9. ${ }^{1} \mathrm{H}$ and ${ }^{13} \mathrm{C}$ date of $\mathbf{P}_{2}$. 

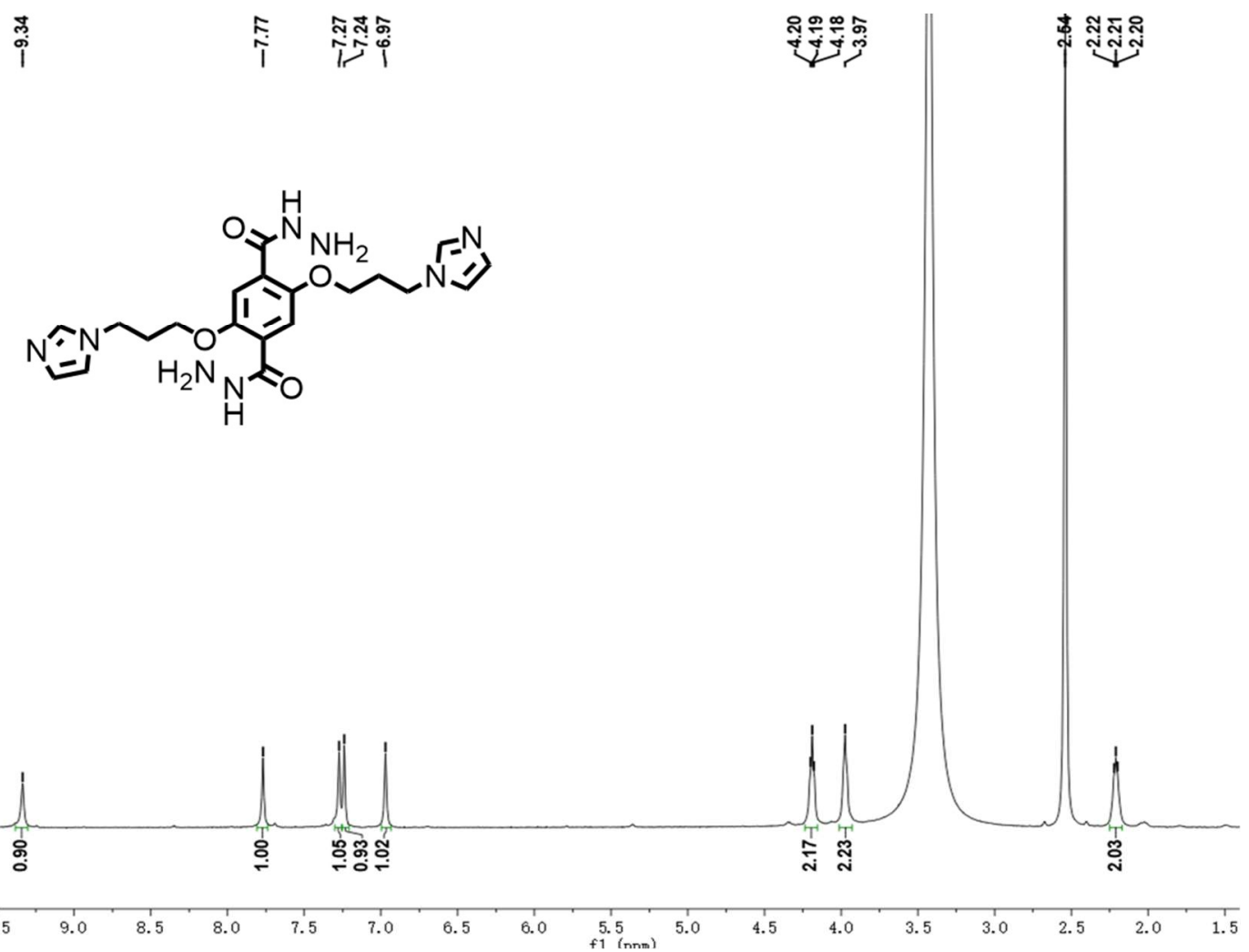

Figure S10. ${ }^{1} \mathrm{H}$ date of $\mathbf{P}_{\mathbf{3}}$.

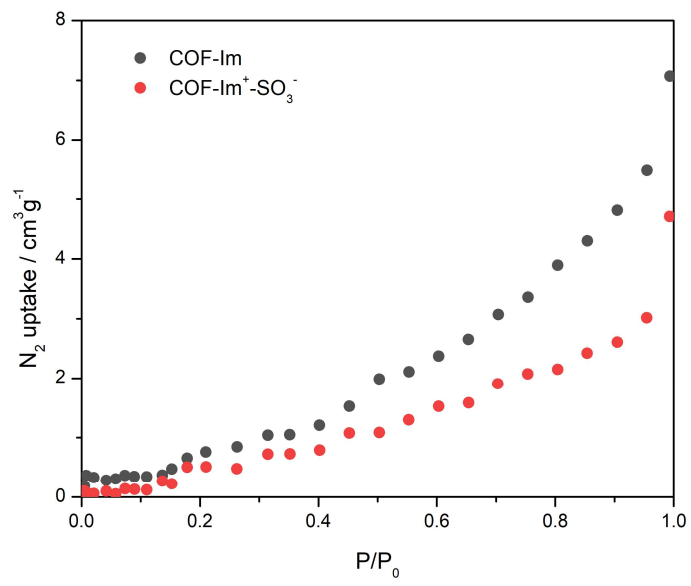

Figure S11. $\mathrm{N}_{2}$ adsorption of COF-Im ${ }^{+}-\mathrm{SO}_{3}{ }^{-}$and $\mathrm{COF}-\mathrm{Im}$. 


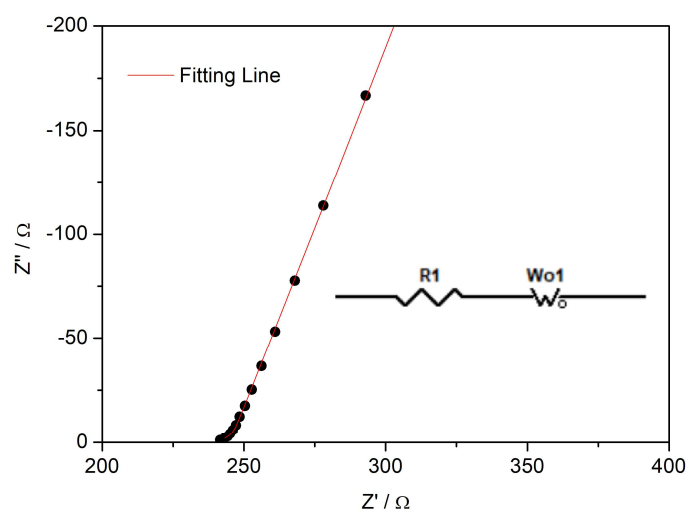

Figure S12. Impedance fitting result of $\mathrm{NH}_{4} \mathrm{Br} @ \mathrm{COF}-\mathrm{Im}^{+}-\mathrm{SO}_{3}{ }^{-}$at $90{ }^{\circ} \mathrm{C}$ and $100 \% \mathrm{RH}$.

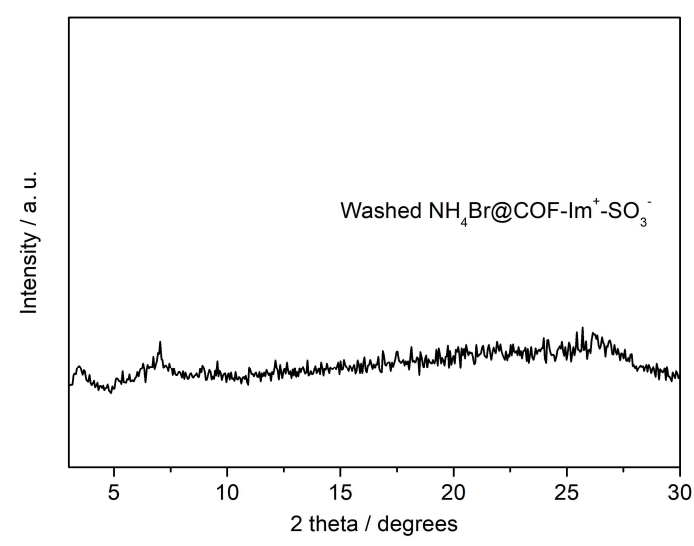

Figure S13. PXRD of washed $\mathrm{NH}_{4} \mathrm{Br} @ \mathrm{COF}-\mathrm{Im}^{+}-\mathrm{SO} 3^{-}$.

\section{References:}

(1) Xue, W. L.; Deng, W. H.; Chen, H.; Liu, R. H.; Taylor, J. M.; Li, Y. K.; Wang, L.; Deng, Y. H.; Li, W. H.; Wen, Y. Y.; Wang, G. E.; Wan, C. Q.; Xu, G., Angew. Chem., Int. Ed. 2021, 60, 1290-1297.

(2) Mu, Z. J.; Ding, X.; Chen, Z. Y.; Han, B. H., ACS Appl. Mater. Interfaces 2018, 10, 41350-41358.

(3) Materials Studio; Accelrys: San Diego. 